\title{
Dual spaces generated by the interior of the set of norm attaining functionals
}

\author{
by
Maria D. Acosta, Julio Becerra Guerrero and
Manuel Ruiz Galán (Granada)

\begin{abstract}
We characterize some isomorphic properties of Banach spaces in terms of the set of norm attaining functionals. The main result states that a Banach space is reflexive as soon as it does not contain $\ell_{1}$ and the dual unit ball is the $w^{*}$-closure of the convex hull of elements contained in the "uniform" interior of the set of norm attaining functionals. By assuming a very weak isometric condition (lack of roughness) instead of not containing $\ell_{1}$, we also obtain a similar result. As a consequence of the first result, a convex-transitive Banach space not containing $\ell_{1}$ and such that the set of norm attaining functionals has nonempty interior is in fact superreflexive.
\end{abstract}

Introduction. James' Theorem states that a Banach space $X$ is reflexive as soon as the set $\mathrm{NA}(X)$ of norm attaining functionals coincides with the dual space $X^{*}$ [13]. A result due to Bourgain and Stegall states that $\mathrm{NA}(X)$ is of first Baire category if $X$ is separable and the unit ball is not dentable (see [5, Problem 3.5.6]). Kenderov, Moors and Sciffer showed the same result for $C(K)$ ( $K$ infinite and compact) [15, Theorem 4].

There are also characterizations of reflexivity by assuming non-empty interior of the set of norm attaining functionals for topologies weaker than the norm topology. For instance, Debs, Godefroy and Saint-Raymond proved that a separable space $X$ such that the $w^{*}$-interior of $\mathrm{NA}(X)$ relative to the dual unit sphere $S_{X^{*}}$ is not empty, is reflexive [6, Lemma 11]. Jiménez Sevilla and Moreno showed the same result for any Banach space $X[14$, Proposition 3.2]. A result by Petunin and Plichko is along the same lines: a separable Banach space $X$ is isometric to a dual space whenever there

2000 Mathematics Subject Classification: 46B10, 46B04.

Research of M. D. Acosta and M. Ruiz Galán partially supported by D.G.E.S., project no. BFM 2000-1467.

Research of J. Becerra Guerrero partially supported by Junta de Andalucía Grant FQM0199. 
is a closed and weak* dense subspace of $X^{*}$ contained in the set of norm attaining functionals [16].

Assuming that $\mathrm{NA}(X)$ has nontrivial (norm) interior, no isomorphic assumption can imply reflexivity of $X$. As a matter of fact, every Banach space can be equivalently renormed so as to have nonempty interior of the set of norm attaining functionals [1]. However, it was proven by Acosta and Ruiz Galán that a separable Banach space $X$ that is very smooth or satisfies the Mazur intersection property has to be reflexive if $\mathrm{NA}(X)$ has nonempty interior [1]. Jiménez and Moreno showed the same result for spaces with the Mazur intersection property without the assumption of separability [14]. Acosta and Ruiz Galán showed that, for a space with Hahn-Banach smooth norm, either $X$ is reflexive or $\mathrm{NA}(X)$ has empty interior [2].

In this note, we get results along the same lines, but assuming an isomorphic hypothesis on the space and a condition stating that the dual unit ball is generated by a subset of the interior of $\mathrm{NA}(X)$ (Theorem 1). We show that any Banach space $X$ can be equivalently renormed so that the dual unit ball is the $w^{*}$-closure of the elements of the unit sphere in the interior of $\mathrm{NA}(X)$.

Afterwards, we prove that an isometric condition (nonroughness of the space) and the generation by the $w^{*}$-closure of the convex hull of the points in the dual unit ball contained in the "uniform" norm interior of $\mathrm{NA}(X)$ also imply reflexivity (Proposition 4). As a consequence, the previous result is valid for any Asplund space. Also we exhibit an example of a space $X$, isomorphic to $c_{0}$ and satisfying a certain condition of differentiability of the norm (weaker than Fréchet), such that $\mathrm{NA}(X)$ has nonempty interior. This example answers in the negative an open question posed in [2].

Results. We will assume that the spaces considered are real, but the results also hold in the complex case and the adaptation of the proofs is immediate.

In the following, $X$ will be a Banach space, $B_{X}$ and $S_{X}$ the closed unit ball and the unit sphere of $X$, respectively. $X^{*}$ will be the topological dual of $X$ and $\mathrm{NA}(X) \subseteq X^{*}$ the subset of norm attaining functionals. For $r>0$ we will write

$$
\mathrm{NA}_{r}(X):=\left\{x^{*} \in X^{*}: x^{*}+r B_{X^{*}} \subset \mathrm{NA}(X)\right\} .
$$

As mentioned in the introduction, no isomorphic condition can imply reflexivity, even if we assume that the set of norm attaining functionals has nonempty interior. Up to now, all the relevant results (except James' Theorem) use an additional isometric assumption. Here we will use an isomorphic condition and a stronger assumption on the set of norm attaining functionals to get a new characterization of reflexivity. 
TheOREM 1. Let $X$ be a Banach space not containing an isomorphic copy of $\ell_{1}$ and assume that for some $r>0$,

$$
B_{X^{*}}=\overline{\mathrm{co}}^{w^{*}}\left\{x^{*} \in S_{X^{*}}: x^{*}+r B_{X^{*}} \subset \mathrm{NA}(X)\right\},
$$

where $\overline{\mathrm{co}} w^{*}$ denotes the $w^{*}$-closure of the convex hull. Then $X$ is reflexive.

Proof. We argue by contradiction. Assume that $X$ is not reflexive. Since $X$ does not contain $\ell_{1}, X$ does not have the Grothendieck property (see [19, Theorem 1] or [12, Proposition 1]). Hence, there exists a $w^{*}$-null sequence $\left\{x_{n}^{*}\right\}$ in $S_{X^{*}}$ which is not weakly null. If $\Phi \in B_{X^{* * *}} \backslash\{0\}$ is a $\sigma\left(X^{* * *}, X^{* *}\right)$ cluster point of $\left\{x_{n}^{*}\right\}$, it is clear that $\Phi(X)=\{0\}$. If $x^{*} \in S_{X^{*}} \cap \mathrm{NA}_{r}(X)$ and $x^{* *} \in S_{X^{* *}}$, we can assume, by passing to a subsequence if necessary, that

$$
x^{* *}\left(x_{n}^{*}\right) \rightarrow \Phi\left(x^{* *}\right) .
$$

Now it follows from Simons' inequality ([7, Lemma I.3.7]) for the functions $f_{n}:=x^{*}+r x_{n}^{*}$ and the sets $B_{X} \subseteq B_{X^{* *}}$ that

$$
x^{* *}\left(x^{*}\right)+r \Phi\left(x^{* *}\right) \leq 1, \quad \forall x^{* *} \in S_{X^{* *}},
$$

that is,

$$
x^{* *}\left(x^{*}\right)+r \Phi\left(x^{* *}\right) \leq\left\|x^{* *}\right\|, \quad \forall x^{* *} \in X^{* *} .
$$

Now, fix $x_{0}^{* *} \in S_{X^{* *}}$ and $\varepsilon>0$. By using again the fact that $X$ does not contain $\ell_{1}$ and [18, Theorem 10] (or [10, Theorem 1]) there are $x_{0} \in S_{X}$ and $\alpha>0$ so that

$$
\operatorname{Osc} x_{0}^{* *}\left(S\left(B_{X^{*}}, x_{0}, \alpha\right)\right)<\varepsilon,
$$

where $S\left(B_{X^{*}}, x_{0}, \alpha\right)=\left\{x^{*} \in B_{X^{*}}: x^{*}\left(x_{0}\right)>1-\alpha\right\}$ and Osc denotes oscillation (i.e. sup - inf).

Since $\left\|x_{0}\right\|=1$, by assumption there is a sequence $\left\{x_{n}^{*}\right\}$ in $X^{*}$ such that

$$
x_{n}^{*}\left(x_{0}\right) \rightarrow 1 \text { and } x_{n}^{*} \in \mathrm{NA}_{r}(X) \cap S_{X^{*}}, \quad \forall n .
$$

Choose a $w^{*}$-cluster point $x^{* * *} \in X^{* * *}$ of $\left\{x_{n}^{*}\right\}$. By (3) we can apply inequality (1) to each $x_{n}^{*}$ and the element $x_{0}+t x_{0}^{* *}$ for any $t>0$, so

$$
x_{n}^{*}\left(x_{0}\right)+t x_{0}^{* *}\left(x_{n}^{*}\right)+r t \Phi\left(x_{0}^{* *}\right) \leq\left\|x_{0}+t x_{0}^{* *}\right\| .
$$

Since $x^{* * *}$ is a $w^{*}$-cluster point of $\left\{x_{n}^{*}\right\}$, it follows from (3) that

$$
1+t x^{* * *}\left(x_{0}^{* *}\right)+r t \Phi\left(x_{0}^{* *}\right) \leq\left\|x_{0}+t x_{0}^{* *}\right\|,
$$

that is,

$$
x^{* * *}\left(x_{0}^{* *}\right)+r \Phi\left(x_{0}^{* *}\right) \leq \frac{\left\|x_{0}+t x_{0}^{* *}\right\|-1}{t}, \quad \forall t>0 .
$$

We have $\lim _{t \rightarrow 0^{+}}\left(\left\|x_{0}+t x_{0}^{* *}\right\|-1\right) / t=\max V\left(x_{0}, x_{0}^{* *}\right)([8$, Theorem V.9.5]), where

$$
V\left(x_{0}, x_{0}^{* *}\right)=\left\{y^{* * *}\left(x_{0}^{* *}\right): y^{* * *} \in S_{X^{* * *}}, y^{* * *}\left(x_{0}\right)=1\right\} .
$$


By Goldstine's Theorem the slice $S\left(B_{X^{*}}, x_{0}, \alpha\right)$ is $w^{*}$-dense in the slice $S\left(B_{X^{* * *}}, x_{0}, \alpha\right)$, so

$$
\operatorname{Osc} x_{0}^{* *}\left(S\left(B_{X^{* * *}}, x_{0}, \alpha\right)\right)=\operatorname{Osc} x_{0}^{* *}\left(S\left(B_{X^{*}}, x_{0}, \alpha\right)\right) .
$$

Since $x^{* * *}$ is a $w^{*}$-cluster point of $\left\{x_{n}^{*}\right\},(3)$ gives us $x^{* * *}\left(x_{0}\right)=1$. By $(2)$, (4) and the previous observation we get

$$
x^{* * *}\left(x_{0}^{* *}\right)+r \Phi\left(x_{0}^{* *}\right) \leq \max V\left(x_{0}, x_{0}^{* *}\right) \leq x^{* * *}\left(x_{0}^{* *}\right)+\varepsilon .
$$

The inequality $r \Phi\left(x_{0}^{* *}\right) \leq \varepsilon$, valid for any $\varepsilon>0$ and $x_{0}^{* *} \in S_{X^{* *}}$, gives $\Phi=0$, a contradiction.

It has been known before that the condition $\mathrm{NA}_{r}(X) \neq \emptyset$ does not suffice to get reflexivity of the space. To show that the first condition imposed $\left(X \not \supset \ell_{1}\right)$ is needed, let us consider the next basic example:

Remark 2. For $X=\ell_{1}$,

$$
B_{X^{*}}=\overline{\mathrm{co}} w^{*}\left(\mathrm{NA}_{1 / 2}(X) \cap S_{X^{*}}\right) .
$$

Proof. Clearly, the convex hull of the points in $S_{X^{*}}$ with finite support is $w^{*}$-dense in $B_{X^{*}}$. Fix one such point $z_{0} \in S_{X^{*}}$. If $z \in S_{X^{*}}$ and $\left\|z-z_{0}\right\| \leq 1 / 2$, then $|z(n)| \leq 1 / 2$ for every $n \notin \operatorname{supp} z_{0}$, while $|z(k)| \geq 1 / 2$ for some $k \in \operatorname{supp} z_{0}$ and therefore $z \in \operatorname{NA}(X)$.

Also, the second assumption imposed in Theorem 1 is needed:

Proposition 3. For any Banach space $Z$, there is a Banach space $X$ isomorphic to $Z$ so that

$$
B_{X^{*}}=\overline{\mathrm{co}}\left(\bigcup_{r>0}\left[\mathrm{NA}_{r}(X) \cap S_{X^{*}}\right]\right) .
$$

Proof. Of course, we can assume that $\operatorname{dim} Z \geq 2$. Let $M$ be a closed linear subspace of $Z$ and $0 \neq z_{0} \in Z$ so that $Z=\mathbb{R} z_{0} \oplus M$ and consider

$$
X=\mathbb{R} z_{0} \oplus_{1} M,
$$

that is, $B_{X}=\operatorname{co}\left\{ \pm z_{0} \cup B_{M}\right\}$ and so

$$
\left|x^{*}\right|=\max \left\{\left|x^{*}\left(z_{0}\right)\right|,\left\|x_{\mid M}^{*}\right\|\right\},
$$

where \|\| is the original norm on $Z$ and | | denotes the new norm.

Define the functional $z_{0}^{*}$ by

$$
z_{0}^{*}\left(z_{0}\right)=1, \quad z_{0}^{*}(m)=0, \quad \forall m \in M .
$$

Clearly, $\left|z_{0}^{*}\right|=1$, and for any $x^{*} \in X^{*}$ with $\left|x^{*}\right| \leq 1$ we have

$$
x^{*}=\frac{1+x^{*}\left(z_{0}\right)}{2}\left(z_{0}^{*}+x^{*} P_{M}\right)+\frac{1-x^{*}\left(z_{0}\right)}{2}\left(-z_{0}^{*}+x^{*} P_{M}\right),
$$

where $P_{M}$ is the natural projection from $X$ to $M$. Since 


$$
\left|z_{0}^{*}+x^{*} P_{M}\right|=\left|-z_{0}^{*}+x^{*} P_{M}\right|=\max \left\{\left|z_{0}^{*}\left(z_{0}\right)\right|,\left\|x_{\mid M}^{*}\right\|\right\}=1,
$$

we have proved that $B_{X^{*}} \subseteq \operatorname{co}\left\{x^{*} \in B_{X^{*}}:\left|x^{*}\left(z_{0}\right)\right|=1\right\}$.

It is enough to approximate each element $x_{0}^{*}$ in $B_{X^{*}}$ satisfying $x_{0}^{*}\left(z_{0}\right)=1$ by functionals in the interior of $\mathrm{NA}(X)$. For such an $x_{0}^{*}$, if we take

$$
x_{n}^{*}=z_{0}^{*}+\left(1-\frac{1}{n}\right) x_{0}^{*} P_{M} \quad(n \in \mathbb{N}),
$$

we get

$$
x_{0}^{*}=\lim _{n} x_{n}^{*} .
$$

Since $\left|x_{n}^{*}\right|=\max \left\{\left|x_{n}^{*}\left(z_{0}\right)\right|,(1-1 / n)\left\|x_{n \mid M}^{*}\right\|\right\}$ and

$$
(1-1 / n)\left\|x_{n \mid M}^{*}\right\| \leq 1-1 / n<1=\left|x_{n}^{*}\left(z_{0}\right)\right|,
$$

the same inequality holds in some open set containing $x_{n}^{*}$. That is, there is an open set of norm attaining functionals containing $x_{n}^{*}$.

By looking carefully at the proof of Theorem 1, one may think that the existence in the dual space of slices with small diameter (a stronger assumption than condition (2) appearing in the proof of Theorem 1) could make a similar result hold. We will get a new result in this direction by assuming that the space is nonrough (instead of not containing $\ell_{1}$ ). First of all, recall that the norm $\|\cdot\|$ on a Banach space is rough if for some $\varepsilon>0$,

$$
\limsup _{h \rightarrow 0} \frac{\|x+h\|+\|x-h\|-2\|x\|}{\|h\|} \geq \varepsilon, \quad \forall x \in X .
$$

Note that Asplund spaces admit no equivalent rough norm.

By assuming a condition on the size of $\mathrm{NA}(X)$ - satisfied by $\ell_{1}$ - and a geometric assumption on $X$, we will get reflexivity.

Proposition 4. A Banach space $X$ is reflexive if, and only if, its norm is not rough and for some $r>0$,

$$
B_{X^{*}}=\overline{\mathrm{CO}} w^{*}\left(\mathrm{NA}_{r}(X) \cap S_{X^{*}}\right) .
$$

Proof. A reflexive space is Asplund, so the norm is not rough and clearly satisfies the second condition since $\mathrm{NA}(X)=X^{*}$. Assume now that the norm of $X$ is not rough and the dual unit ball can be generated by $\mathrm{NA}_{r}(X)$ as above. [7, Proposition I.1.11] yields $x \in S_{X}$ and $\alpha>0$ satisfying

$$
\operatorname{diam} S\left(B_{X^{*}}, x, \alpha\right)<r,
$$

where $S\left(B_{X^{*}}, x, \alpha\right)$ is the $w^{*}$-slice given by

$$
S\left(B_{X^{*}}, x, \alpha\right)=\left\{x^{*} \in B_{X^{*}}: x^{*}(x)>1-\alpha\right\} .
$$

By using the second assumption and the definition of $S\left(B_{X^{*}}, x, \alpha\right)$, it follows that $S\left(B_{X^{*}}, x, \alpha\right)$ contains some element $x^{*} \in S_{X^{*}} \cap \mathrm{NA}_{r}(X)$. There- 
fore,

$$
S\left(B_{X^{*}}, x, \alpha\right) \subset x^{*}+r B_{X^{*}} \subset \mathrm{NA}(X)
$$

and by [14, Lemma 3.1], $X$ is reflexive.

Corollary 5. $X$ is reflexive if, and only if, the following conditions are satisfied:

(i) $X$ is Asplund or at least its norm is Fréchet differentiable at some point.

(ii) There is $r>0$ such that

$$
B_{X^{*}}=\overline{\mathrm{co}}^{w^{*}}\left(\mathrm{NA}_{r}(X) \cap S_{X^{*}}\right) .
$$

It is known that Banach spaces $X$ with the Mazur intersection property such that $\mathrm{NA}(X)$ has nonempty interior, are reflexive [14, Proposition 3.3]. In Proposition 4 we assumed a weaker condition on the space and a stronger assumption on $\mathrm{NA}(X)$.

Note that there are nonreflexive Asplund spaces $X$ with $\mathrm{NA}(X)$ having nonempty interior. In fact, we will give an example of a space $X$ isomorphic to $c_{0}$ such that the interior of $\mathrm{NA}(X)$ is not empty and the norm of $X$ satisfies a certain differentiability condition. Recall that the norm $\|\cdot\|$ on a Banach space $X$ is strongly subdifferentiable (see $[9,11]$ ) at a point $u \in S_{X}$ if

$$
\lim _{\alpha \rightarrow 0^{+}} \frac{\|u+\alpha x\|-1}{\alpha}=\tau(u, x) \quad \text { uniformly for } x \in B_{X} .
$$

A smooth norm satisfying the previous condition at any point $u$ of the unit sphere is Fréchet differentiable indeed.

The following example also answers in the negative a question posed in [2, Open Question 1].

EXAMPlE 6 . There is a Banach space $X$ isomorphic to $c_{0}$ so that the norm of $X$ is strongly subdifferentiable (at any point of $S_{X}$ ) and $\mathrm{NA}(X)$ has nonempty interior. Therefore, $X$ is not reflexive but Asplund.

Proof. It is enough to take as $X$ the space $c_{0}$ endowed with the equivalent norm $\|\cdot\|$ given by

$$
\|x\|:=\frac{1}{2}|x(1)|+\max _{n \geq 2}|x(n)| \quad\left(x \in c_{0}\right) .
$$

This norm is strongly subdifferentiable as both the summands are.

If $\left\{e_{n}\right\}$ is the usual basis of $c_{0}$, we consider the open set in $X^{*}$ given by

$$
O=\left\{x^{*} \in X^{*}: 2\left|x^{*}\left(e_{1}\right)\right|>\sum_{n=2}^{\infty}\left|x^{*}\left(e_{n}\right)\right|\right\} .
$$


For $x^{*} \in O$ and $x \in X$ we have

$$
\begin{aligned}
\left|x^{*}(x)\right| & =\left|\sum_{n=1}^{\infty} x^{*}\left(e_{n}\right) x(n)\right| \\
& \leq\left|x^{*}\left(e_{1}\right) x(1)\right|+\sum_{n=2}^{\infty}\left|x^{*}\left(e_{n}\right)\right| \max _{n \geq 2}|x(n)| \\
& \leq 2\left|x^{*}\left(e_{1}\right)\right|\left(\frac{1}{2}|x(1)|+\max _{n \geq 2}|x(n)|\right) \\
& \leq 2\left|x^{*}\left(e_{1}\right)\right|\|x\|,
\end{aligned}
$$

hence $\left\|x^{*}\right\| \leq 2\left|x^{*}\left(e_{1}\right)\right|$. Since $\left\|2 e_{1}\right\|=1$, we have $\left\|x^{*}\right\|=2\left|x^{*}\left(e_{1}\right)\right|$ and $x^{*}$ attains its norm, therefore $\mathrm{NA}(X)$ has nonempty interior.

The presence of points with special properties in the interior of $\mathrm{NA}(X)$ and the nonroughness of $X$ force reflexivity. In order to be more precise, we recall some definitions.

For a Banach space $X$, let $\mathcal{G}$ be the group of all surjective linear isometries on $X$. The space $X$ is said to be convex-transitive if $B_{X}=\overline{\operatorname{co}} \mathcal{G}(u)$ for any $u \in S_{X}$. The spaces $L_{1}[0,1], L_{\infty}[0,1]$ and the Calkin algebra are convextransitive (see [17, Theorem 9.6.4] and [4, Corollary 4.6]).

Our aim now is to exhibit a class of Banach spaces $X$ which are superreflexive in the case where the interior of $\mathrm{NA}(X)$ is nonempty.

Proposition 7. Let $X$ be a separable Banach space, which is convextransitive and has $\mathrm{NA}(X)$ of second Baire category (in the norm topology). Then $X$ is superreflexive.

Proof. Since $X$ is separable and $\mathrm{NA}(X)$ is of second Baire category, the unit ball of $X$ is dentable (see [5, Theorem 3.5.5 and Problem 3.5.6]). By [7, Proposition I.1.11], this implies that the norm of $X^{*}$ is not rough, and by using the convex-transitivity of $X, X$ is superreflexive in view of $[3$, Theorem $3.2]$.

Proposition 8. Let $X$ be a convex-transitive Banach space not containing $\ell_{1}$ such that $\mathrm{NA}(X)$ has nonempty interior. Then $X$ is superreflexive.

Proof. Since $\mathrm{NA}(X)$ is a cone with nonempty interior, there is $x^{*} \in S_{X^{*}}$ so that $x^{*}+r B_{X^{*}} \subset \mathrm{NA}(X)$ for some $r>0$. That is, $x^{*} \in \mathrm{NA}_{r}(X) \cap S_{X^{*}}$. As $X$ is convex-transitive, we have

$$
B_{X^{*}}=\overline{\mathrm{co}}^{w^{*}}\left(\bigcup_{T \in \mathcal{G}} T^{*} x^{*}\right) .
$$

Now, $x^{*}+r y^{*} \in \mathrm{NA}(X)$ for any $y^{*} \in B_{X^{*}}$, so there is $y \in S_{X}$ satisfying

$$
\left|\left(x^{*}+r y^{*}\right)(y)\right|=\left\|x^{*}+r y^{*}\right\| \text {. }
$$


Then, for any $T \in \mathcal{G}$,

$$
T^{*}\left(x^{*}+r y^{*}\right)\left(T^{-1}(y)\right)=\left(x^{*}+r y^{*}\right)(y)=\left\|x^{*}+r y^{*}\right\|=\left\|T^{*}\left(x^{*}+r y^{*}\right)\right\|
$$

and $\left\|T^{-1}(y)\right\|=\|y\|=1$, that is, $T^{*}\left(x^{*}+r y^{*}\right) \in \mathrm{NA}(X)$. Since the above condition holds for any $y^{*} \in B_{X^{*}}$, and $T^{*}$ is a surjective isometry on $X^{*}$, we have $T^{*} x^{*} \in S_{X^{*}} \cap \mathrm{NA}_{r}(X)$. By using (1) it follows that

$$
B_{X^{*}}=\overline{\mathrm{co}}^{w^{*}}\left(\mathrm{NA}_{r}(X) \cap S_{X^{*}}\right) \text {. }
$$

Since we are assuming that $X$ does not contain $\ell_{1}$, in view of Theorem 1 , $X$ is reflexive. Also, by [3, Theorem 3.2], since $X$ is convex-transitive, it is, in fact, superreflexive.

The previous positive results suggest the following question:

Open problem 9. Let $X$ be a convex-transitive Banach space such that $\mathrm{NA}(X)$ has nonempty interior. Is $X$ superreflexive?

Acknowledgements. The second author is grateful to Angel Rodríguez Palacios for valuable suggestions.

\section{References}

[1] M. D. Acosta and M. Ruiz Galán, New characterizations of the reflexivity in terms of the set of norm attaining functionals, Canad. Math. Bull. 41 (1998), 279-289.

[2] - - - Norm attaining operators and reflexivity, Rend. Circ. Mat. Palermo 56 (1998), 171-177.

[3] J. Becerra Guerrero and A. Rodríguez Palacios, The geometry of convex-transitive Banach spaces, Bull. London Math. Soc. 31 (1999), 323-331.

[4] -, - , Transitivity of the norm on Banach spaces having a Jordan structure, Manuscripta Math. 102 (2000), 111-127.

[5] R. D. Bourgin, Geometric Sspects of Convex Sets with the Radon-Nikodym Property, Lecture Notes in Math. 993, Springer, Berlin, 1983.

[6] G. Debs, G. Godefroy and J. Saint Raymond, Topological properties of the set of norm-attaining linear functionals, Canad. J. Math. 47 (1995), 318-329.

[7] R. Deville, G. Godefroy and V. Zizler, Smoothness and Renormings in Banach Spaces, Pitman Monographs Surveys Pure Appl. Math. 64, Longman Sci. Tech., New York, 1993.

[8] N. Dunford and J. T. Schwartz, Linear Operators Part I: General Theory, Interscience Publ., New York, 1958.

[9] C. Franchetti and R. Payá, Banach spaces with strongly subdifferentiable norm, Boll. Un. Mat. Ital. B 7 (1993), 45-70.

[10] J. R. Giles, Comparable differentiability characterisations of two classes of Banach spaces, Bull. Austral. Math. Soc. 56 (1997), 263-272.

[11] G. Godefroy, V. Montesinos and V. Zizler, Strong subdifferentiability of norms and geometry of Banach spaces, Comment. Math. Univ. Carolin. 36 (1995), 493-502.

[12] M. González and J. M. Gutiérrez, Polynomial Grothendieck properties, Glasgow Math. J. 37 (1995), 211-219.

[13] R. C. James, Weak compactness and reflexivity, Israel J. Math. 2 (1964), 101-119. 
[14] M. Jiménez Sevilla and J. P. Moreno, A note on norm attaining functionals, Proc. Amer. Math. Soc. 126 (1998), 1989-1997.

[15] P. S. Kenderov, W. B. Moors and S. Sciffer, Norm attaining functionals on $C(T)$, ibid. 126 (1998), 153-157.

[16] Yu. I. Petunin and A. N. Plichko, Some properties of the set of functionals that attain a supremum on the unit sphere, Ukrain. Mat. Zh. 26 (1974), 102-106 (in Russian); MR 49:1075.

[17] S. Rolewicz, Metric Linear Spaces, Math. Appl. 20, Reidel, Dordrecht, 1985.

[18] E. Saab and P. Saab, A dual geometrical characterization of Banach spaces not containing $\ell_{1}$, Pacific J. Math. 105 (1983), 415-425.

[19] M. Valdivia, Fréchet spaces with no subspaces isomorphic to $\ell_{1}$, Math. Japon. 38 (1993), 397-411.

Maria D. Acosta

Departamento de Análisis Matemático

Facultad de Ciencias

Universidad de Granada

18071 Granada, Spain

E-mail: dacosta@ugr.es
Julio Becerra Guerrero and Manuel Ruiz Galán Departamento de Matemática Aplicada

E.U. Arquitectura Técnica

Universidad de Granada 18071 Granada, Spain E-mail: juliobg@ugr.es mruizg@ugr.es

Received February 20, 2001

Revised version June 11, 2001 